\title{
Pigmented Conjunctival Lesions as Presenting Signs of Vaginal Melanoma
}

\author{
Paul O. Phelps ${ }^{\text {a }}$ Martha J. Farber ${ }^{\text {b }}$ Dale R. Meyer ${ }^{\text {a }}$ \\ ${ }^{a}$ Ophthalmic Plastic Surgery, Lions Eye Institute-Albany Medical Center, Slingerlands, NY, and \\ ${ }^{b}$ Albany Stratton Veterans Affairs Medical Center, Albany, NY, USA
}

\section{Keywords}

Melanoma · Conjunctival neoplasms · Vaginal neoplasms .

Neoplasm metastasis

\begin{abstract}
A 43-year-old woman was referred to an oculoplastic surgeon for the evaluation of 2 small pigmented lesions in the tarsal conjunctiva. She underwent a biopsy of the lesions, both of which were found to be consistent with metastatic melanoma. Follow-up with the patient revealed that she had been hospitalized for balance issues and headaches. An MRI of her brain revealed a hemorrhagic mass and several smaller foci suspicious for metastatic lesions. A thorough workup included an evaluation for bloody vaginal discharge. Biopsy of a pigmented portion of the vaginal mucosa lesion revealed BRAF-positive melanoma. This was determined to be the primary site of her malignant metastatic melanoma, which caused her death within 6 months of presentation.
\end{abstract}

(c) 2017 S. Karger AG, Basel

The development of a pigmented lesion of the conjunctiva may rightly raise clinical suspicion for primary acquired melanosis or melanoma. These lesions are often particularly suspicious in patients with a light complexion, or when present on the tarsal or forniceal conjunctiva. Rarely, this type of lesion may represent an even more sinister underlying condition. There are multiple reports in the literature of cutaneous melanomas metastasizing to the conjunctiva, and one large case series reported 2 of these cases [1]. We report the first documented case of pigmented conjunctival lesions as the presenting signs of vaginal melanoma.

\section{Case Report}

A 43-year-old woman was referred to an oculoplastic surgeon for the evaluation of 2 small pigmented lesions of the conjunctiva (Fig. 1a). The patient underwent a biopsy of the lesions, which were both consistent with metastatic melanoma (Fig. 1b, c). Follow-up with the patient revealed that she had been hospitalized for balance issues and headaches. An MRI of her brain revealed a hemorrhagic mass and several smaller foci suspicious for metastatic lesions. The patient was also evaluated for recent history of bloody vaginal discharge. An ultrasound revealed a mass within the vaginal cavity. Biopsy of the vaginal mucosal mass revealed BRAFpositive melanoma. A thorough oncologic workup determined that the vaginal mass was the primary site of her malignant metastatic melanoma. Despite aggressive chemotherapy and radiation, the metastatic disease led to her death within 6 months of presentation. An autopsy was not performed.

\section{KARGER}

(c) 2017 S. Karger AG, Basel

E-Mail karger@karger.com

www.karger.com/oop
Paul O. Phelps, MD

Ophthalmic Plastic Surgery, Lions Eye Institute-Albany Medical Center

1220 New Scotland Road, Suite 302

Slingerlands, NY 12159 (USA)

E-Mail pophelps@gmail.com 

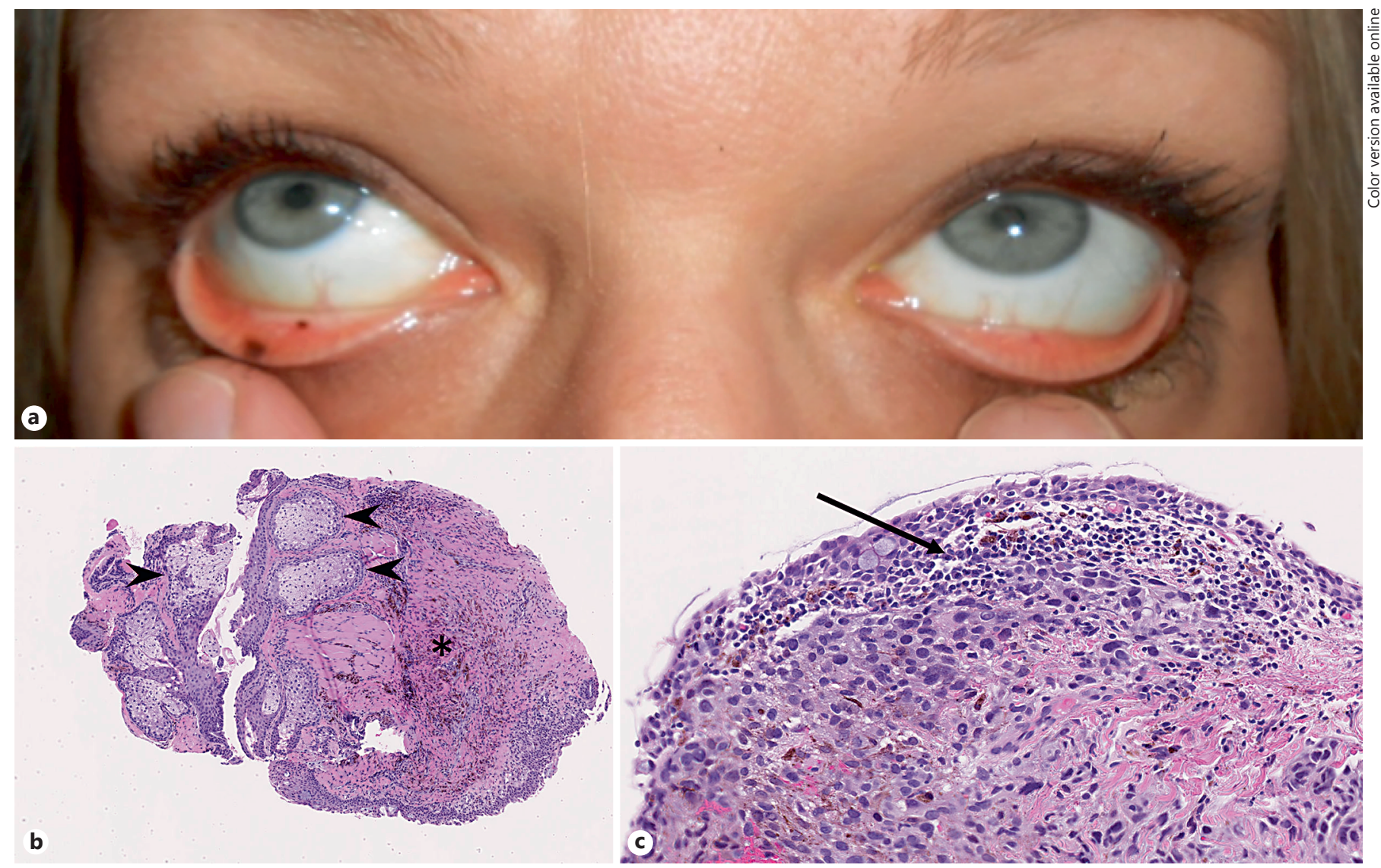

Fig. 1. A 43-year-old woman presented with 2 pigmented lesions of her tarsal conjunctiva (a). The patient reported that she first noticed the lesions 2 months prior. Biopsy of the lesions revealed pigmented melanocytes with atypia and large nucleus-to-cytoplasm ratio. A low-magnification view (b) demonstrated meibo-

mian gland architecture (arrowheads) with pigmented cells extending into the substantia propria (asterisk). A high-magnification view (c) revealed melanocytes with anaplastic features and surrounding lymphocytic inflammation (arrow).

\section{Discussion}

The pigmented conjunctival lesions in our case did not demonstrate aggressive clinical features such as feeder vessels, varied pigmentation, or irregular borders typical of conjunctival melanoma. Histopathology did not demonstrate intraepithelial proliferation of abnormal melanocytes with variable atypia. These features would have been more consistent with conjunctival melanoma arising from primary acquired melanosis. Several clock hours of primary acquired melanosis along the limbus was also not present, a finding that has been shown to correlate with conjunctival melanoma [2]. The lesions also lacked the cysts typical of conjunctival nevus. An unusual feature was the presence of 2 separate lesions of the palpebral conjunctiva, which arose de novo.

Vaginal melanoma has a very low cure rate, even with radical surgery and chemotherapy, with a 5 -year survival rate of $11.4 \%$ for female genital tract melanoma [3]. Interestingly, the vaginal wall biopsy in our case demonstrated BRAF-positive melanoma. Mutations in BRAF have been shown to be significantly more common in acral and mucous membrane melanomas than those involving chronically sun-damaged skin [4]. However, one study of genetic alterations in vulvar and vaginal melanomas did not identify a case of BRAF-positive melanoma [5]. In our case, the patient developed rapid progression of her disease, with hematogenous spread to her brain, lungs, and liver. Her melanoma did not demonstrate lymphatic involvement. Hematogenous spread is common for mucosal melanomas, specifically vaginal melanoma [6]. This contrasts with cutaneous melanomas, which 
typically spread by a lymphogenous route. The patient was treated with radiation for her brain lesions as well as chemotherapy with dabrafenib (BRAF-targeted therapy) as well as trametinib (MEK inhibitor). She appeared somewhat improved after 2 months of therapy. However, despite aggressive treatment, the patient succumbed to the sequelae of her disease.

The common causes of melanocytic lesions of the conjunctiva are nevus, primary acquired melanosis, benign acquired melanosis, and malignant melanoma. While more than $99 \%$ of pigmented conjunctival lesions are derived from one of those 4 categories [1], it is important for the ophthalmologist to consider metastasis when presented with multiple de novo pigmented conjunctival lesions. It is also essential to have a primary care provider and/or dermatologist involved in the care of these patients. We would consider early biopsy for de novo pig- mented conjunctival lesions given their potential to identify an ominous underlying condition. Our case is the first, to our knowledge, to demonstrate pigmented lesions of the conjunctiva as the presenting signs of vaginal melanoma.

\section{Statement of Ethics}

The collection and evaluation of protected patient health information was HIPPA-compliant. The subject of this case report gave informed consent for inclusion, and the study was exempt from institutional review due to its retrospective nature.

\section{Disclosure Statement}

There are no conflicts of interest to declare.

\section{References}

Conjunctival Metastasis as Presenting Signs of Vaginal Melanoma
1 Shields CL, Demirci H, Karatza E, Shields JA: Clinical survey of 1,643 melanocytic and nonmelanocytic conjunctival tumors. Ophthalmology 2004;111:1747-1754.

2 Shields JA, Shields CL, Mashayekhi A, et al: Primary acquired melanosis of the conjunctiva: experience with 311 eyes. Trans Am Ophthalmol Soc 2007;105:61-71; discussion 71-72.

3 Janco JM, Markovic SN, Weaver AL, Cliby WA: Vulvar and vaginal melanoma: case series and review of current management options including neoadjuvant chemotherapy. Gynecol Oncol 2013;129:533-537.
4 Curtin JA, Fridlyand J, Kageshita T, et al: Distinct sets of genetic alterations in melanoma. N Engl J Med 2005;353:2135-2147.

5 Aulmann S, Sinn HP, Penzel R, et al: Comparison of molecular abnormalities in vulvar and vaginal melanomas. Mod Pathol 2014;27: 1386-1393.

6 O'Regan K, Breen M, Ramaiya N, et al: Metastatic mucosal melanoma: imaging patterns of metastasis and recurrence. Cancer Imaging 2013;13:626-632. 